\title{
Perfil das publicações da área de Ciências Biológicas e da Saúde em revistas brasileiras de extensão universitária
}

\author{
Cristina Berger Fadel ${ }^{1}$, Carlos Machado Filho ${ }^{2}$, Danielle Bordin ${ }^{3}$, Manoelito Ferreira Silva Junior ${ }^{4}$
}

Resumo: O objetivo deste estudo foi descrever o perfil das publicações da área de Ciências Biológicas e da Saúde em revistas de extensão universitária brasileiras. O estudo documental descritivo foi realizado com a totalidade de sítios eletrônicos de revistas de extensão universitária em atividade no Brasil indexados no Sistema Regional de Informação em Linha para Revistas Científicas da América Latina, Caribe, Espanha e Portugal (LATINDEX), por meio de um recorte temporal de dez anos (2008-2017). Foram encontradas 29 revistas extensionistas, sendo a maioria concentradas na região Sul (41,4\%), editoradas por Instituições de Ensino Superior públicas (89,6\%) e predominantemente localizadas no interior $(55,2 \%)$. O periódico mais antigo iniciou sua atividade em 1997, e 2013 foi o ano com o maior número de revistas iniciadas. As revistas foram mais avaliadas no Qualis B3 e C pela Coordenação de Aperfeiçoamento de Pessoal de Nível Superior (CAPES), e foram mais avaliadas na área Interdisciplinar $(93,1 \%)$ e menos na Medicina Veterinária $(34,5 \%)$. Mais da metade das revistas $(55,1 \%)$ publicou até 20 artigos por ano, com frequência predominantemente semestral (75,9\%). Foram publicados 1.231 artigos, sendo a área Interdisciplinar com a maior proporção (21,0\%), e com menor proporção a área de Farmácia (1,8\%). O tema mais presente foi 'Educação em saúde' $(14,0 \%)$. Houve uma expansão no número de revistas e publicações extensionistas universitárias brasileiras, porém com disparidade regional e baixa qualificação dos periódicos. As publicações nas áreas de Ciências Biológicas e da Saúde foram sobre ações preventivas, educativas e de promoção da saúde.

Palavras-chave: Relações Comunidade-Instituição; Revisão por pares; Revistas Eletrônicas; Publicação Periódica; Análise Espaço-Temporal

\section{Profile of publications in the area of Biological and Health Sciences in Brazilian university extension journals}

Abstract: The objective of the study was to describe the profile of publications in the area of Biological and Health Sciences in Brazilian university extension journals. The descriptive documentary study was carried out with all the websites of university extension journals active in Brazil indexed in the Regional Online Information System for Scientific Journals in Latin America, the Caribbean, Spain, and Portugal (LATINDEX), through a ten-year time frame (2008-2017). Twentynine extension journals were found, most of them concentrated in the South region (41.4\%), edited by public Higher Education Institutions ( $89.6 \%$ ), and located in the countryside (55.2\%). The journals started their activity in 1997, and most were created in 2013. The journals were more evaluated in Qualis B3 and C by the Coordination for the Improvement of Higher Education Personnel (CAPES), being more evaluated in the Interdisciplinary area (93.1\%) and more minor in Veterinary Medicine (34.5\%). Most journals published between 11 and 20 (37.9\%) articles with semiannual frequency (75.9\%). Regarding the number of articles, 1,231 were published, the majority in the Interdisciplinary area (21.0\%) and lower proportion in the Pharmacy area (1.8\%). The most present theme was 'Health education' (14.0\%). There was an expansion in the number of Brazilian university extension journals and publications, but with regional disparity and low qualification of journals. Publications in Biological and Health Sciences are more about preventive, educational, and health promotion actions.

Keywords: Community-Institutional Relations; Peer review; Electronic Journals; Periodical; SpaceTime Analysis

\section{Originais recebidos em}

03 de agosto de 2020

Aceito para publicação em

08 de março de 2021

1

Doutora em Odontologia Preventiva e Social. Departamento de Odontologia, Universidade Estadual de Ponta Grossa UEPG. Ponta Grossa, Paraná.

cbfadel@gmail.com

2 Residente em Cirurgia Bucomaxilofacial, Universidade Estadual de Ponta Grossa (UEPG), Paraná.

carlosmachado-55@hotmail.com

3

Professora Colaboradora. Doutora em Odontologia Preventiva e Social. Departamento de Enfermagem e Saúde Pública UEPG. Ponta Grossa, Paraná. Email:

daniellebordin@hotmail.com

4

Professor Colaborador. Doutor em Odontologia (Saúde Coletiva). Departamento de Odontologia UEPG. Ponta Grossa, Paraná.

manoelito fsjunior@hotmail.com (autor para correspondência) 


\section{Introdução}

No Brasil, como em outros países, até meados dos anos 80 a extensão universitária apresentava caráter secundário dentro das universidades e, por isso, apresentava crescimento limitado, embora, eventualmente, surgissem manifestações em sua defesa, principalmente por parte dos estudantes. Ao final da década de 80, houve uma retomada, e um destaque maior na prática extensionista, advindos em parte da queda de financiamento das universidades em diversos países, e de uma crise de legitimidade, caracterizada pelo questionamento ao isolamento academicista (Souza Santos, 2010).

Ao longo da história das universidades brasileiras, e em especial das instituições públicas, a extensão universitária vem perpassando por diversas matizes e diretrizes conceituais. De um processo da extensão dos cursos à extensão dos serviços, à extensão assistencial, à extensão 'redentora da função social da universidade', à via de mão dupla entre universidade e sociedade e à extensão cidadã. Tem sido possível identificar uma ressignificação da extensão nas relações internas com os outros fazeres acadêmicos, e na sua relação com a comunidade (Cristofoletti \& Serafim, 2020). Essa conjunção de interfaces é ainda mais presente e identificada nas áreas do campo de saber da educação e da saúde, onde em natureza, o exercício prático dos cursos tem natureza extramural.

Em 1998, o Fórum Nacional de Pró-Reitores de Extensão das Universidades Públicas Brasileiras (FORPROEX) elaborou o Plano Nacional de Extensão Universitária, no qual se definiu a extensão universitária como o processo educativo, cultural e científico que articula o ensino e a pesquisa de forma indissociável, e viabiliza a relação transformadora entre universidade e sociedade (Fórum de Pró-Reitores de Extensão das Universidades Públicas Brasileiras [FORPROEX], 2001). Sob uma perspectiva crítica, emancipadora e cidadã, o FORPROEX, enquanto instância colegiada, apresentou objetivos distintos e destacou-se em possibilitar novos meios e processos de produção, inovação e transferência de conhecimentos, permitindo a ampliação do acesso ao saber e o desenvolvimento tecnológico e social do país.

A discussão conceitual e prática que gere a questão do conhecimento passa então a ser considerada na interface da extensão, ensino e pesquisa, visando a integralidade da formação acadêmica. Na dimensão pedagógica, a extensão passa a ser, portanto, uma estratégia de busca ou justificativa de maiores investimentos e, ao mesmo tempo, promoção de uma legitimação do saber acadêmico na direção das demandas sociais, tornando-o mais aplicada, e buscando sua validação em situações mais tangíveis (Coelho, 2014). No entanto, questiona-se ainda se esse objetivo almejado tem sido incorporado na prática dentro das instituições, e se medidas foram realizadas para garantir a visibilidade que a extensão universitária faz jus e necessita.

A divulgação científica, por meio de periódicos e a publicação de artigos, pode ser um indicador no processo de sistematizar e potencializar as atividades realizadas no campo da extensão universitária (Sidone et al., 2016; Landim et al., 2017; Cristofoletti \& Serafim, 2020). O presente artigo se debruça sobre as publicações impressas brasileiras exclusivas em periódicos universitários extensionistas, e tem por objetivo descrever o perfil das publicações da área de Ciências Biológicas e da Saúde em revistas de extensão universitária brasileiras.

\section{Metodologia}

Trata-se de pesquisa documental de caráter descritivo, realizada a partir da análise da totalidade de sítios eletrônicos de revistas de extensão universitária em atividade no Brasil, considerando um recorte temporal de dez anos, entre janeiro de 2008 a dezembro de 2017. 
Para a localização das revistas extensionistas, foram realizadas buscas em bases de indexação no Sistema Regional de Informação em Linha para Revistas Científicas da América Latina, Caribe, Espanha e Portugal (LATINDEX, 2018) e consulta via contato telefônico a pró-Reitores ligados ao Fórum de Pró-Reitores de Extensão das Instituições Públicas de Educação Superior Brasileiras (FORPROEX), para ratificação de informações de interesse.

Como critério de inclusão foram consideradas somente revistas com publicações de práticas, reflexões e resultados de ações de extensão nas grandes áreas das Ciências Biológicas e da Saúde, englobando os espaços multiprofissionais (com integração entre duas ou mais áreas da saúde).

As variáveis pesquisadas em foram: distribuição geográfica do periódico com potencial de difusão do conhecimento, instituição mantenedora e cidade do periódico, área e número de artigos encontrados, concentração de artigos publicados por revista, periodicidade de publicação das edições, classificação Qualis dos periódicos conforme a Plataforma Sucupira - Coordenação de Aperfeiçoamento de Pessoal de Nível Superior (CAPES) por área de avaliação no quadriênio 2013-2016, e tema principal de publicação, sendo possível cada periódico apresentar avaliação em 48 áreas diferentes. Nesta avaliação, houve uma variação de oito estratos de do WebQualis, indo do estrato mais elevado para o de menos qualificação, na seguinte ordem: A1, A2, B1, B2, B3, B4, B5, C (Barata, 2016). Em relação aos temas publicados, todos foram analisados por um dos autores, e os termos de sintaxe mais próxima foram vinculados em único tema, nominado 'tema principal', para facilitar a compreensão e interpretação dos achados.

Após tabulação dos dados em planilha eletrônica, houve síntese quantitativa, através de estatística descritiva, com frequência absoluta (n) e relativa (\%). Um mapa foi construído para ilustrar a distribuição das publicações no Brasil.

\section{Resultados}

Foram encontradas um total de 29 revistas extensionistas universitárias indexadas em bases de dados brasileiras de acesso aberto (Listadas na Tabela S1, Material Suplementar). No intervalo temporal de dez anos, foram encontrados um total de 1.231 artigos publicados na área das Ciências Biológicas e da Saúde, sendo a metade concentrada em quatro periódicos.

As revistas são mantidas em sua totalidade por Instituições de Ensino Superior (IES), sendo a maioria de natureza pública (89,6\%): universidades federais $(n=16)$, estaduais $(n=7)$ e institutos federais $(n=3)$. Entre as IES particulares $(10,4 \%)$, duas eram universidades comunitárias e uma sem fins lucrativos. Ainda, a maioria delas eram pertencentes à IES localizadas em municípios do interior (55,2\%).

Em relação à distribuição geográfica das revistas, as mesmas encontram-se distribuídas em 14 unidades federativas brasileiras, percebendo-se uma maior concentração de revistas na região Sul (41,4\%), sendo mais presentes no Rio Grande do Sul e Santa Catarina (Figura 1). A segunda maior concentração de revistas ocorreu na região Sudeste (27,6\%), nos estados de Minas Gerais e São Paulo. O Nordeste, apesar da baixa concentração de revistas (17,2\%), abriga a revista de maior difusão nacional, um periódico do Rio Grande do Norte, que detém $21,5 \%$ de todas as publicações no período analisado. As regiões Centro-Oeste e Norte em conjunto contribuíram com $13,8 \%$ das revistas de extensão universitárias brasileiras.

Verificou-se que a revista mais antiga iniciou suas publicações no ano de 1997, e o ano de 2013 apresentou o maior número de revistas iniciadas ( $n=7)$ (Figura 2).

A área Interdisciplinar foi a que apresentou a maior proporção de revistas com publicações $(93,1 \%)$ e a área de Medicina Veterinária foi a que apresentou a menor proporção, com publicações em apenas $34,5 \%$ das 
revistas. Quanto ao número de artigos publicados, novamente a área Interdisciplinar apresentou a maior proporção (21,0\%), e a área de Farmácia a menor (1,8\%) (Tabela 1).

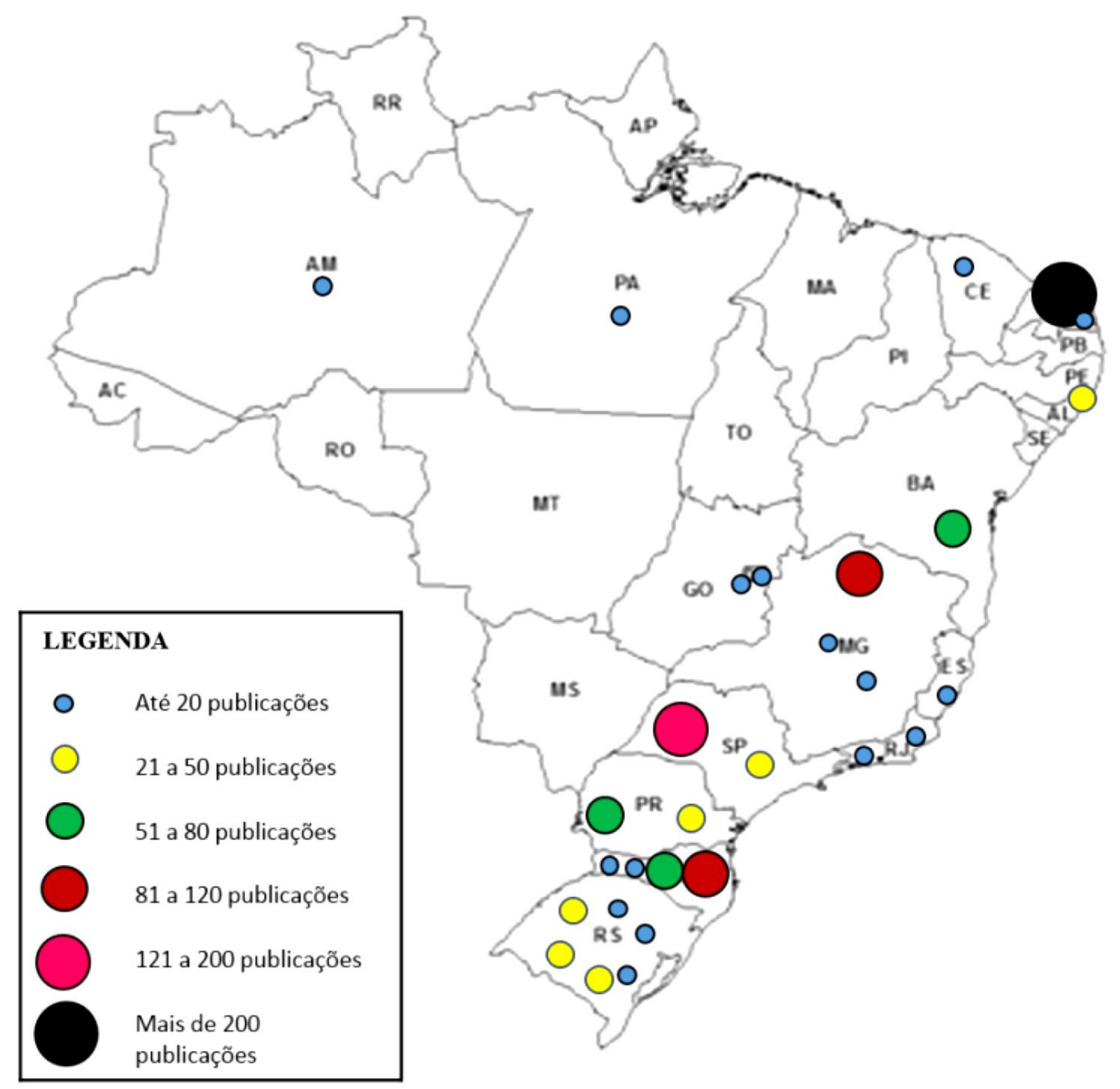

Figura 1. Concentração das revistas de extensão e seus respectivos potenciais de difusão do conhecimento, de acordo com o número de itens publicados, por unidade federativa brasileira, no período de 2008 a 2017.

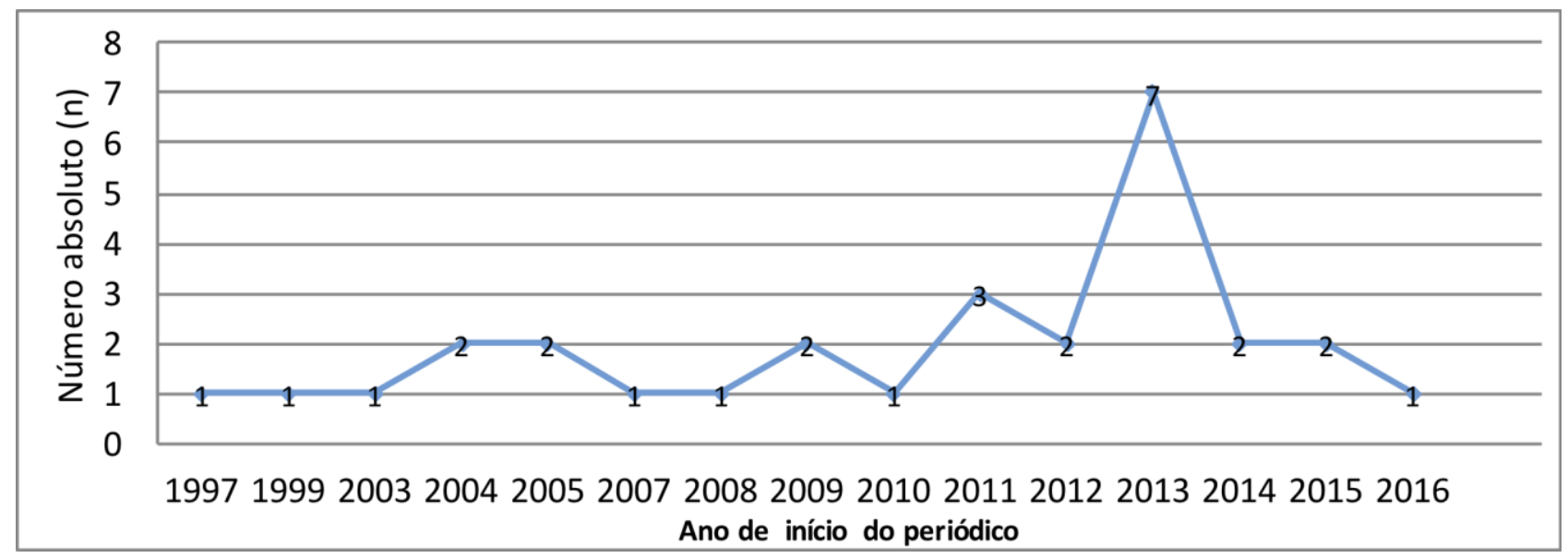

Figura 2. Distribuição por ano do início dos periódicos extensionistas brasileiros identificados no levantamento. 
Tabela 1. Perfil de revistas de extensão universitária, segundo área e número de artigos publicados, no período de 2008 a 2017 ( $n=29)$. Brasil, 2017.

\begin{tabular}{lcc}
\hline Área & $\begin{array}{c}\text { Número de revistas com artigos } \\
\text { publicados por área - } \mathrm{n}(\%)^{*}\end{array}$ & $\begin{array}{c}\text { Artigos publicados por área } \\
-\mathrm{n}(\%)\end{array}$ \\
\hline Interdisciplinar & $27(93,1)$ & $259(21,0)$ \\
\hline Enfermagem & $24(82,8)$ & $227(18,4)$ \\
\hline Biologia & $23(79,3)$ & $139(11,3)$ \\
\hline Medicina & $23(79,3)$ & $102(8,3)$ \\
\hline Odontologia & $21(72,4)$ & $251(20,4)$ \\
Nutrição & $19(65,5)$ & $70(5,7)$ \\
\hline Educação Física & $17(58,6)$ & $86(7,0)$ \\
Farmácia & $13(44,8)$ & $22(1,8)$ \\
Fisioterapia & $11(37,9)$ & $48(3,9)$ \\
Medicina Veterinária & $10(34,5)$ & $28(2,3)$ \\
\hline Total & $29(100,0)$ & $1.231(100,0)$ \\
\hline
\end{tabular}

* Uma revista pode publicar em mais de uma área de inserção. A porcentagem se refere, neste caso, ao número total de revistas encontradas.

Segundo a classificação Qualis CAPES, as revistas apresentaram qualificação entre B3 e C nas diversas áreas de avaliação inseridas nas grandes áreas da Saúde e Ciências Biológicas. Um total de três revistas não apresentaram classificação para nenhuma das áreas descritas (Tabela 2).

No período analisado, a maioria das revistas apresentou entre 11 e 20 (37,9\%) números publicados, com predomínio de frequência semestral (75,9\%) (Tabela 3). Observou-se um aumento da frequência de publicação, de anual para semestral, quadrimestral ou trimestral para uma parcela de revistas ( $n=8,27,6 \%$ ), e uma revista reduziu a sua frequência de semestral para anual $(3,4 \%)$.

Dos 1.231 artigos publicados, os temas mais abordados na área das Ciências Biológicas e da Saúde foram relacionados à 'Educação em saúde' ( $n=172,14,0 \%)$ e 'Educação ambiental' ( $n=143,11,6 \%)$, e os menos abordados: 'Formação profissional' ( $n=3,0,2 \%)$ e 'Gestão em saúde' ( $n=1,0,1 \%$ ) (Figura 3).

\section{Discussão}

Apesar de que ainda são encontradas poucas revistas extensionistas no Brasil, como demonstrado em outros estudos (Coelho, 2014; Landim et al., 2017), ficou evidente o crescimento do número de periódicos nas últimas décadas (Coelho, 2014). Um estudo anterior demonstrou um processo de desenvolvimento científico no Brasil, nas diversas áreas e, entre os triênios avaliados, as Ciências da Saúde e Ciências Biológicas foram as áreas que mais produziram artigos entre 1992-1994 e 2007-2009 (Sidone et al., 2016). Esse dado corrobora os resultados do presente estudo, delimitado à extensão universitária, observando-se também uma contribuição e número expressivo de publicações nessas áreas. Acredita-se que este aspecto possa decorrer da indissociabilidade 
cada vez maior entre o ensino nas áreas em questão (Ciências Biológicas e da Saúde) e a prestação de serviços de saúde, estreitamente vinculados ao processo de ensino-aprendizagem (Cristofoletti \& Serafim, 2020).

Tabela 2. Perfil de revistas de extensão, segundo classificação na Capes por área, no período de 2008 a $2017(n=29)$. Brasil, 2017.

\begin{tabular}{lccccc}
\hline & \multicolumn{5}{c}{ Qualis Capes dos Periódicos* } \\
Área** & B3 & \multicolumn{1}{c}{ B4 } & \multicolumn{1}{c}{ B5 } & \multicolumn{1}{c}{ C } & Sem Qualis*** (\%) \\
\hline Ciências Biológicas & $0(0,0)$ & $0(0,0)$ & $0(0,0)$ & $7(24,1)$ & $22(75,9)$ \\
Educação Física & $0(0,0)$ & $5(17,2)$ & $3(10,3)$ & $0(0,0)$ & $21(72,4)$ \\
Enfermagem & $0(0,0)$ & $10(34,5)$ & $5(17,2)$ & $0(0,0)$ & $14(48,3)$ \\
Farmácia & $0(0,0)$ & $0(0,0)$ & $0(0,0)$ & $2(6,9)$ & $27(93,1)$ \\
Interdisciplinar & $3(10,3)$ & $9(31,0)$ & $8(27,6)$ & $1(3,4)$ & $7(24,1)$ \\
Medicina & $0(0,0)$ & $0(0,0)$ & $3(10,3)$ & $6(20,7)$ & $20(69,0)$ \\
Medicina Veterinária & $0(0,0)$ & $0(0,0)$ & $4(13,8)$ & $0(0,0)$ & $25(86,2)$ \\
Nutrição & $0(0,0)$ & $0(0,0)$ & $1(3,4)$ & $0(0,0)$ & $28(96,6)$ \\
Odontologia & $0(0,0)$ & $11(37,9)$ & $2(6,9)$ & $0(0,0)$ & $16(55,2)$ \\
\hline
\end{tabular}

*Avaliação do quadriênio 2013-2016.

**Uma revista pode publicar em mais de uma área de inserção.

*** Nas áreas de avaliação de Ciências Biológicas ou da Saúde.

Tabela 3. Perfil de revistas de extensão, segundo concentração de artigos publicados por revista e periodicidade de publicação, no período de 2008 a 2017 (n=29). Brasil, 2017.

\begin{tabular}{|c|c|c|}
\hline & & evistas - n (\%) \\
\hline \multirow{8}{*}{$\begin{array}{l}\text { Número de artigos por revista } \\
\text { (concentração) }\end{array}$} & Até 10 & $5(17,2)$ \\
\hline & 11 a 20 & $11(37,9)$ \\
\hline & 21 a 40 & $3(10,3)$ \\
\hline & 41 a 60 & $4(13,8)$ \\
\hline & 61 a 80 & $2(6,9)$ \\
\hline & 81 a 100 & $2(6,9)$ \\
\hline & 101 a 200 & $1(3,4)$ \\
\hline & Mais de 200 & $1(3,4)$ \\
\hline \multirow{4}{*}{ Periodicidade de publicação } & Trimestral & $1(3,4)$ \\
\hline & Quadrimestral & $2(6,9)$ \\
\hline & Semestral & $22(75,9)$ \\
\hline & Anual & $4(13,8)$ \\
\hline
\end{tabular}




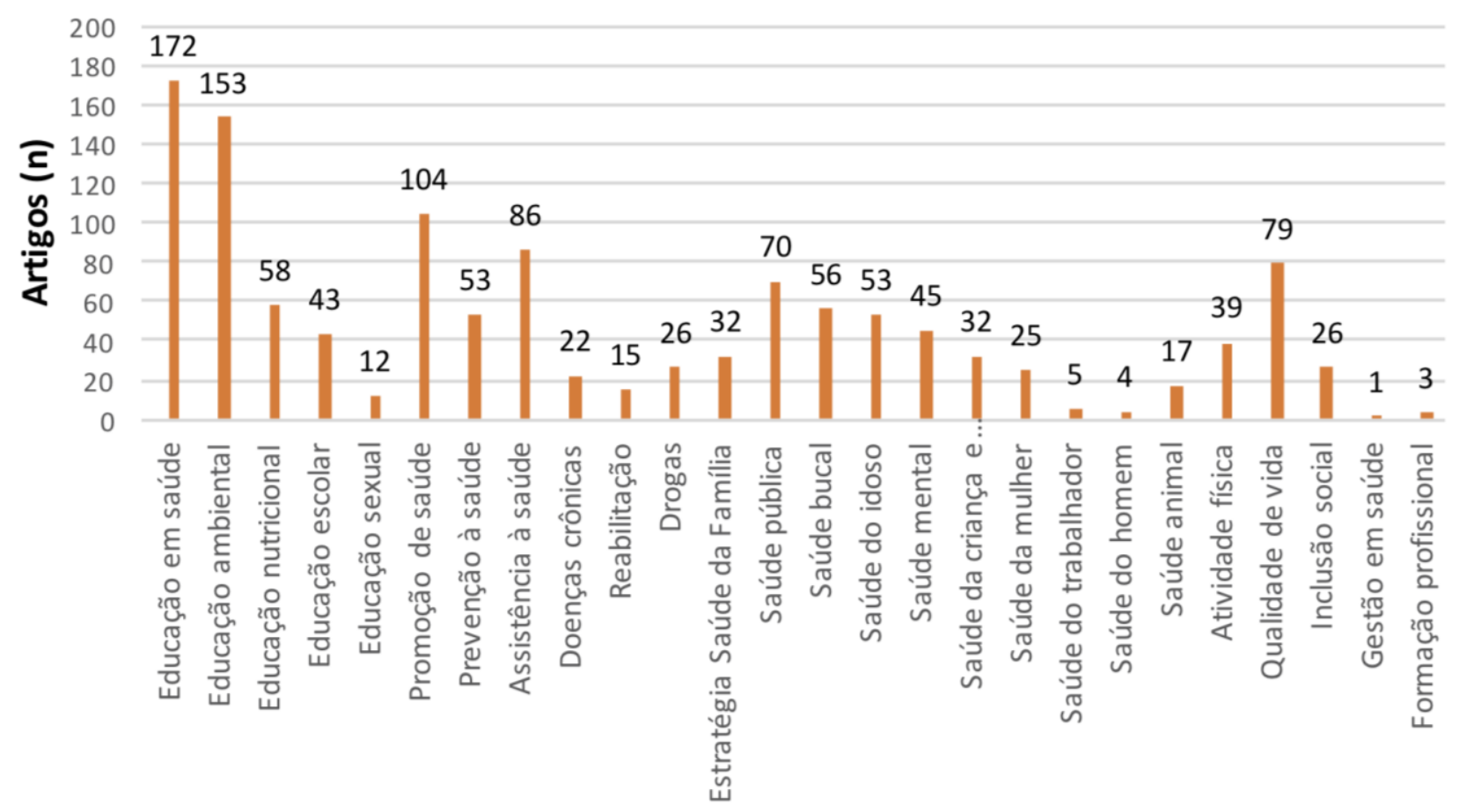

Temas abordados

Figura 3. Perfil de revistas de extensão, segundo valor absoluto do tema principal de publicação, no período de 2008 a 2017 ( $n=1.231)$. Brasil, 2017.

No presente estudo, verificou-se disparidades regionais na distribuição dos periódicos extensionistas no Brasil, como também demonstrado em estudo prévio (Coelho, 2014). Essa distribuição mantem a lógica de concentração das universidades brasileiras (Barros, 2015), no que se refere à produção científica brasileira em geral (Sidone et al., 2016), ou seja, mais acentuada nas regiões mais desenvolvidas do país, Sul e Sudeste.

No entanto, tem-se percebido um processo lento, mas gradual, de expansão das redes de colaboração e aumento da participação de autores de regiões cientificamente menos tradicionais, como o Nordeste (Sidone et al., 2016). Inclusive, um periódico do Nordeste destacou-se pelo grande número de publicações, concentrando quase $1 / 4$ de todos os artigos publicados nas revistas extensionistas no período analisado neste estudo. Neste sentido, deve-se ressaltar a necessidade de políticas públicas que favoreçam o desenvolvimento extensionista e o incentivo às atividades científicas e, consequentemente, o desenvolvimento das regiões menos favorecidas (Sidone et al., 2016).

As revistas extensionistas brasileiras encontradas iniciaram suas atividades no final da década de 90 . Apesar da literatura apontar a existência de periódicos extensionistas no Brasil desde a década de 80 (Coelho, 2014), tais como: Revista Cadernos, da Pró-Reitoria de Extensão da Pontifícia Universidade Católica de Minas Gerais, Revista Desafio, da Universidade Federal do Ceará, ambas de 1988, e ainda a Revista de Extensão da Universidade Federal da Paraíba, de 1996, estas não estão ativas atualmente. Sendo assim, houve um crescimento de novos periódicos durante os anos 2000, e um crescimento mais expressivo no ano de 2013. 
Esse aspecto pode derivar de políticas públicas de expansão, valorização e o processo de interiorização do ensino superior público no Brasil (Costa, 2017).

No presente estudo, a maioria dos periódicos extensionistas encontrados são mantidos e administrados por instituições públicas, como verificado nos demais periódicos brasileiros (Souza et al., 2018), o que reforça o valor atribuído ao ensino superior público brasileiro, para além do viés do ensino formal. As IES impulsionam o desenvolvimento regional, ao empoderar os indivíduos, por meio da qualificação cientifica, e na capacidade de resolver demandas da sociedade com uso e progresso tecnológico, e melhora da eficácia na produção (Sousa \& Freiesleben, 2018). Esse aspecto poderia explicar, em parte, que a maioria dos periódicos avaliados serem administrados por IES localizados em municípios do interior do país, ou seja, onde há maior interação entre o meio acadêmico e a população e, com isso, maior desenvolvimento loco-regional (Oliveira-Junior, 2014). Sendo assim, as universidades no interior podem apresentar maior responsabilidade e preocupação em desenvolver estudos e dar maior visibilidade às experiências extensionistas, e assim, ter maior interesse em editorar periódicos nessa perspectiva da extensão universitária.

Quanto à periodicidade das revistas, a maioria apresentou publicação semestral. Estudos anteriores demonstraram dificuldades no processo editorial das revistas científicas, principalmente relacionado ao corpo editorial, periodicidade (Coelho, 2014) e indexação em bases de dados, na busca da qualificação e aumento do alcance das publicações (Landim et al., 2017). Apesar disso, no presente estudo, verificou-se uma tendência de aumento de números por volume (ano) e de artigos por número (fascículo) publicado. Esse dado torna-se relevante ao considerar que, além do incremento anual de novos periódicos, houve maior número de artigos por ano, o que aumenta a capacidade de produção e divulgação cientifica em extensão no Brasil. No entanto, o crescimento foi lento, e pode ainda ser consequência da baixa qualificação dos periódicos extensionistas ou ainda, do método empregados na investigação. Um estudo demonstrou que a maioria dos artigos presentes nos periódicos extensionistas são relatos de experiência, e poucos são oriundos de pesquisas advindas da extensão universitária, ou de temas que possam subsidiá-la (Coelho, 2014).

Dada a indiscutível importância da extensão universitária, o baixo Qualis dos periódicos extensionistas pode ainda permitir que os pesquisadores brasileiros reflitam se vale a pena o gasto de recursos humanos e financeiros para a publicação de dados cuja temática ou periódico sejam voltadas para a extensão universitária. Além disso, um estudo mostrou que o processo de seleção para carreira docente em magistério superior público apresenta uma menor relevância ao item de extensão, ou seja, os docentes ainda precisam demonstrar maior experiência no âmbito do ensino e da pesquisa. Além disso, após o ingresso docente, o mesmo estudo apontou que a importância da prática da extensão no desempenho da carreira acadêmica ainda é frágil, uma vez que se pode progredir e atingir o nível mais alto da carreira sem ter atuado na extensão (Teleginski, 2018).

A maioria dos periódicos analisados apresentava artigos na área Interdisciplinar, sendo incluídos nessa categoria apenas os artigos publicados que envolviam diretamente conteúdo nas áreas das Ciências Biológicas ou da Saúde. Este resultado demonstra uma mudança no paradigma na formação profissional, com projetos de extensão que têm buscado cada vez mais uma interface de interlocução entre as áreas do conhecimento e, assim, uma valorização da atuação interdisciplinar como forma de obtenção de melhores resultados e de melhoria da qualidade de vida da população (Azevedo, 2017).

Os artigos no campo das Ciências Biológicas e da Saúde nos periódicos avaliados abordaram experiências exitosas com temas relacionados aos benefícios da educação, prevenção e promoção da saúde, e buscavam a melhoria dos determinantes do processo saúde-doença. Vale ressaltar que a integração ensino-serviçocomunidade traz consigo a necessidade de valorização de práticas de prevenção e promoção de saúde, em um cuidado integral de saúde, com o uso da educação em saúde como meio de transformação social (Faé et al., 2016). 
O presente estudo mostrou a importância da manutenção e expansão das revistas extensionistas como forma de incentivo e divulgação de atividades extramurais e dos seus benefícios, tanto para a formação profissional, quanto para o desenvolvimento da comunidade, impactando positivamente a saúde pública no Brasil. Esperase que, em médio prazo, haja uma expansão quantitativa e qualitativa das revistas extensionistas do Brasil, como meio de estimular e aprimorar a disseminação de práticas extensionistas.

\section{Conclusão}

Apesar de poucas revistas extensionistas universitárias brasileiras, verificou-se um processo de expansão do número dos periódicos, da periodicidade das revistas e de publicação da publicação de artigos, principalmente no ano de 2013.

Os periódicos são na maioria editorados por instituições de ensino superior de natureza pública, e alocados no interior do país, embora com disparidades regionais, e maior concentração de revistas nas regiões Sul e Sudeste.

Verificou-se ainda uma baixa qualificação para os periódicos extensionistas, segundo a avaliação da CAPES, especialmente nas áreas das Ciências Biológicas e da Saúde. Nestas áreas, houve maior identificação de publicações de artigos sobre ações preventivas, educativas e de promoção de saúde.

\section{Contribuição de cada autor}

O autor C.M.F. realizou a coleta dos dados; C.B.F., D.B. e M.F.S.J. contribuíram com as análises estatísticas e escreveram o texto final; C.B.F. atuou como coordenadora e orientadora do bolsista.

\section{Referências}

Azevedo, A. B., Pezzato, L. M., \& Mendes, R. (2017). Formação interdisciplinar em saúde e práticas coletivas. Saúde em Debate, 41(113), 647-657.

Barata, R. B. (2016). Dez coisas que você deveria saber sobre o Qualis. Revista Brasileira de Pós-Graduação, 13(30), 1340.

Barros, A. S. X. (2015). Expansão da educação superior no Brasil: Limites e possibilidades. Educação \& Sociedade, 36(131), 361-390.

Coelho, G. C. (2014). Revistas acadêmicas de extensão universitária no Brasil. Revista Brasileira de Extensão Universitária, 5(2), 69-75.

Costa, M. R. S. (2017). Contextualizando expansão e interiorização no campo da educação brasileira. Revista Exitus, 7(1), 250-276.

Cristofoletti, E. C., \& Serafim, M. P. (2020). Dimensões Metodológicas e Analíticas da Extensão Universitária. Educação e Realidade, 45(1), e90670.

Landim, T. C. F., Matos, B. G., \& Chagas, R. M. V. (2017). A indexação das revistas de extensão: A experiência brasileira. Extensão em Ação, 2(14), 32-43.

Oliveira-Junior, A. (2014). A Universidade como polo de desenvolvimento local-regional. Caderno de Geografia, 24 (Spec 1), 1-12. 
Fórum de Pró-Reitores de Extensão das Universidades Públicas Brasileiras (2001). Plano Nacional de Extensão Universitária, Edição Atualizada. [S. I.]: FORPROEX/ Secretaria de Educação Superior do Ministério da Educação e do Desporto. Recuperado de http://www.prae.ufrpe.br/sites/p rae.ufrpe.br/files/pnextensao 1.pdf

Sidone, O. J. G., Haddad, E. A., \& Mena-Chalco, J. P. (2016). A ciência nas regiões brasileiras: Evolução da produção e das redes de colaboração científica. TransInformação, 28(1), 15-31.

Faé, J. M., Silva-Junior, M.F, Carvalho, R. B., Esposti, C. D. D., \& Pacheco, K. T. S. (2016). A integração ensino-serviço em Odontologia no Brasil. Revista da ABENO, 16(3), 7-18.

Sistema Regional de Informação em Linha para Revistas Científicas da América Latina, Caribe, Espanha e Portugal (2018). Catálogo. Cidade do México: LATINDEX. Recuperado de https://www.latindex.org/latindex/tablaPais?id=9\&id2=1

Sousa, F. E., \& Freiesleben, M. (2018). A educação como fator de desenvolvimento regional. Revista da FAE, 21(2), 163178.

Sousa Santos, B. Universidade no século XXI: Para uma reforma democrática e emancipatória da Universidade. 3. ed. São Paulo: Cortez, 2010.

Souza C. D., Filippo, D., \& Casado, E. S. (2018). Crescimento da atividade científica nas universidades federais brasileiras: Análise por áreas temáticas. Avaliação - Revista da Avaliação da Educação Superior, 23(1), 126-156.

Teleginski, D. E. (2018). A relevância da extensão nos processos de seleção e de avaliação de desempenho de docentes da universidade contemporânea: Casos da UTFPR e Unicamp (Dissertação de Mestrado). Universidade Tecnológica Federal do Paraná, Curitiba, Brasil. Recuperado de http://repositorio.utfpr.edu.br/jspui/handle/1/3789

Como citar este artigo:

Fadel, C. B., Machado Filho, C., Bordin, D., \& Silva Junior, M. F. (2021). Perfil das publicações da área de Ciências Biológicas e da Saúde em revistas brasileiras de extensão universitária. Revista Brasileira de Extensão Universitária, 12(1), 125-134. https://periodicos.uffs.edu.br/index.php/RBEU/ article/view/11652/pdf 